\title{
ON LAMÉ OPERATORS WHICH ARE PULL-BACKS OF HYPERGEOMETRIC ONES
}

\author{
BRUNO CHIARELLOTTO
}

\begin{abstract}
We give a method that would allow one to calculate the number of Lamé operators, $\mathscr{L}_{n}, n \in \mathbf{N}$, with prescribed finite monodromy and do the calculation for the case $n=1$. We find a bound for the degree over $\mathbf{Q}$ of the field of definition of the coefficients of a Lamé operator with prescribed finite monodromy and give examples of Lamé operators with finite monodromy. Finally we study Lamé operators with infinite monodromy and generic second order differential operators which are pull-backs of hypergeometric ones under algebraic maps.
\end{abstract}

Consider an ordinary differential operator

$$
L=\frac{d^{n}}{d x^{n}}+a_{1}(x) \frac{d^{n-1}}{d x^{n-1}}+\cdots+a_{n}(x)
$$

where $a_{i}(x) \in K(x)$ and $K$ is a number field over $\mathbf{Q}$ : what one can say about its solutions $(L y=0)$ and monodromy looking only at the coefficients $a_{i}(x)$ ?

In this paper we give some partial answers to this question for the special case of a Lamé operator. One of the questions we will approach is the following:

(*) For every $n \in \mathbf{N}$, how many Lamé operators $\mathscr{L}_{n}$ are there whose monodromy group is a dihedral group of order $2 N(N \in \mathbf{N})$ ?

We will answer $(*)$ in the case $n=1(2.14,2.15)$. The method we use for the proof works for general $n$ : for example the case $n=2$ can be found in [CO].

Before summarizing our work we give a brief review of the known results.

Since the work of Schwarz on hypergeometric equations and the classification of the relative finite monodromy groups, no basic improvements have been made. Recently, the challenge of the Grothendieck conjecture has stimulated some progress: see [KA], [KA1], [KA2], [HO], [BE-HE], [DW1]. (We recall that this conjecture asserts that if in $(0.1)$ almost all the reductions $\bmod \wp$ (prime ideal in the ring of integers of $K$ ) have a complete set of solutions, which is equivalent to $L$ having zero $\wp$-curvature for almost all $\wp$, then equation $(0.1)$ has only algebraic solutions.) On the other hand, some improvements in the sense of Klein and Schwarz can be found in [BA-DW], [SING], [BA], [DW].

But we are still a long way from achieving the same level of understanding for general operators that we have attained in the hypergeometric case. In

Received by the editors January 2, 1991.

1991 Mathematics Subject Classification. Primary 34B30; Secondary 14E20.

The author was a visiting fellow at Princeton University, supported by C.N.R. Italy. 
particular, this is true for the most obvious extension, namely for a second order differential operator with four singular points.

In this paper we want to deal with a particular case: the Lamé operators, that is, the family of second order differential operators, with four singular points, defined for each $n \in \mathbf{N}^{*}$

$$
\mathscr{L}_{n}=D^{2}+\frac{f^{\prime}}{2 f} D-\frac{n(n+1) x+B}{f}
$$

where $D=\frac{d}{d x}, f=4 \prod_{i=1}^{3}\left(x-e_{i}\right), e_{1} \neq e_{2} \neq e_{3} \neq e_{1}$ and $B, e_{i} \in \mathbf{C}$, and with exponent differences:

$$
\begin{gathered}
\Delta_{\mathscr{L}}\left(e_{i}\right)=\frac{1}{2}, \quad i=1,2,3, \\
\Delta_{\mathscr{L}}(\infty)=n+\frac{1}{2}
\end{gathered}
$$

([PL], [WW]). For such operators the Grothendieck conjecture was proved in $[\mathrm{CH}-\mathrm{CH}]$. But the problem of connecting the parameters $e_{1}, e_{2}, e_{3}, B$ and $n$ with the solutions and the monodromy is still open (see [BA]). As we said before, in this paper we address this problem and, in particular, we will answer the question $(*)$ for $n=1$ up to homography $(2.14,2.15)$.

Remark 0.2. Two Lamé operators $\mathscr{L}_{n}^{\prime}, \mathscr{L}_{n}^{\prime \prime}$ are homographic if one is transformed into the other by a homographic change of the independent variable. For $n \in \mathbf{N}$, a dihedral group is the only possible finite monodromy group for $\mathscr{L}_{n}$ [BA]. We will show that the number (up to homography) of equations for each $n \in \mathbf{N}$ and each $N \in \mathbf{N}$ is finite (we recall that for a Lamé operator finite monodromy is equivalent to having algebraic solutions).

It is known [BA-DW] that every Lamé operator $\mathscr{L}_{n}$ with finite monodromy group of order $2 N$ is a rational pull-back of a hypergeometric one of the type $\left(\frac{1}{2}, \frac{1}{N}, \frac{1}{2}\right)$ ([BA-DW], [BA1]); this kind of hypergeometric operator will be indicated by $\mathscr{H}_{N}$ and we recall that it has the dihedral group of order $2 N$ as monodromy group. If we consider the rational map by which we construct the pull-back as a covering:

$$
\Phi: \mathbf{P}^{1} \rightarrow \mathbf{P}^{1}
$$

the problem $(*)$ becomes related with the calculation of all the rational coverings of $\mathbf{P}^{1}$ (up to homography) with ramification over 0,1 and $\infty$ which transform by pull-back a hypergeometric operator into a Lamé one. This will give the method to solve $(*)$.

Remark 0.3. Following [BA-DW] by pull-back we mean "weak" pull-back (see Remark 1.4).

Once we know the number (up to homography) of the Lamé operators $\mathscr{L}_{n}$ with prescribed monodromy group, we can investigate the number field in which it is possible to define the coefficients. In $\S 3$ we will give a bound for the degree over $\mathbf{Q}$ of this field (Proposition 3.1). This will be done by studying the coefficients of the rational function giving the Lamé $\mathscr{L}_{n}$ as pull-back of a hypergeometric operator.

In [DW1] Dwork conjectures that all globally nilpotent second order operators are related to hypergeometric ones by pull-backs via algebraic maps and by linear transformations of the solutions (leaving the monodromy unchanged). 
In the final part of this paper $(\S 5)$ we will deal with Lamé operators with $n=-\frac{1}{2}$ (and so with infinite monodromy). In $\S 5$ we will investigate (Proposition 5.5) the structure of the algebraic maps which transform (via pull-back) a hypergeometric operator into a generic second order linear operator with rational coefficients.

The plan of the paper is as follows: in $\S 1$ problem $(*)$ is reduced to a covering problem. In $\S 2$ we will answer $(*)$ in the case of Lamé operators with $n=1$ $(2.14,2.15)$ (the same techniques can be applied to the general case: an example is found in [CO]). In $\S 3$ we apply our previous results in order to define a bound for the degree over $\mathbf{Q}$ of the field of definition of the coefficients of a Lamé operator with given $n$ and prescribed dihedral group (Proposition 3.1). In $\S 4$ we give some examples. In $\S 5$ using our method we study Lamé operators with $n=-\frac{1}{2}$ which are rational pull-backs of hypergeometrics (see [CH-CH1, 2]). In this section we also state some results about pull-backs made by algebraic maps. This is a first (small) step in the direction of the Dwork conjecture ([DW1], [CH-CH1, 2]).

We wish to express our gratitude to Professor Bernard Dwork: his patience in introducing us to the subject and his advice have been invaluable. Thanks are also due to C. Falcolini and to Professor J. H. Conway.

1

For notation see [BA-DW], [BA], [DW]. Let $n \in \mathbf{N}^{*}$; we denote by

$$
L_{n}
$$

the set of Lamé operators

$$
\mathscr{L}_{n}=D^{2}+\frac{f^{\prime}}{2 f} D-\frac{n(n+1) x+B}{f}
$$

with $f=4 \prod_{i=1}^{3}\left(x-e_{i}\right), e_{1} \neq e_{2} \neq e_{3} \neq e_{1}, e_{i}, B \in \mathrm{C}$. The four singular points for (1.2) are $e_{i}, e_{2}, e_{3}, \infty$ with exponent differences:

$$
\begin{gathered}
\Delta_{\mathscr{L}_{n}}\left(e_{i}\right)=\frac{1}{2}, \quad i=1,2,3, \\
\Delta_{\mathscr{L}_{n}}(\infty)=n+\frac{1}{2} .
\end{gathered}
$$

For Lamé operators $\mathscr{L}_{n}$ as in (1.2) the dihedral group is the only possible monodromy finite group [BA]. For a given dihedral group of order $2 N, N \in$ $\mathbf{N}^{*}$, we want to know (up to homography $(0.2)$ ) the number of Lamé operators, $\mathscr{L}_{n}$ (1.2), which have this group as monodromy group.

In general, by [BA-DW], such a Lamé operator will be a pull-back by a rational map

$$
\Phi: \mathbf{P}^{1} \rightarrow \mathbf{P}^{1}
$$

of a hypergeometric one

$$
\mathscr{H}_{N}=L_{\frac{1}{2}, \frac{1}{N}, \frac{1}{2}}
$$

which also has a finite dihedral monodromy group of order $2 N$. (The exponent differences of $\mathscr{H}_{N}$ are $\Delta_{\mathscr{H}}(0)=\Delta_{\mathscr{H}}(\infty)=\frac{1}{2}$, and $\Delta_{\mathscr{H}}(1)=\frac{1}{N} ;$ cf. (1.11).) 
Remark 1.4. Take the second order differential equation

$$
\mathscr{L}_{n} Y=0 \text {. }
$$

The Klein theory of pull-backs is connected with the ratio of the solutions [BADW]: it follows that transformations of the type $Y=\theta Z$ (with $\theta$ locally defined) are allowed and the operators $\mathscr{L}_{n}$ and $\frac{1}{\theta} \circ \mathscr{L}_{n} \circ \theta$ are considered equivalent. By this type of transformation on $\mathscr{L}_{n}$ one can locally reduce it to the form

$$
D^{2}+B^{\prime}
$$

where $B^{\prime}=-\frac{n(n+1) x+B}{f}-\frac{1}{4} \frac{f^{\prime \prime}}{f}+\frac{3}{16}\left(\frac{f^{\prime}}{f}\right)^{2}$ and $\theta$ is defined as $\frac{\theta^{\prime}}{\theta}=-\frac{1}{4} \frac{f^{\prime}}{f}$. (Of course from (1.4.1) one can recover $\mathscr{L}_{n}$.) The notion of weak pull-back is related to operators in this normalized form (see [BA-DW]). But (1.4.1) has the same singularities and the same exponent differences as $\mathscr{L}_{n}$ and it uniquely characterizes the $\mathscr{L}_{n}$ by which it has been generated: this implies that homographic equivalence for (1.4.1)-type operators is equal to the same equivalence for the operators $\mathscr{L}_{n}$ from which they derive. On the other hand, an easy calculation shows that no transformations (except the constant one) of the type $\frac{1}{\theta} \circ \mathscr{L}_{n} \circ \theta$ send $\mathscr{L}_{n}$ into another element of $L_{n}$. It follows that our notion is perfectly consistent with [BA-DW], [BA1]. See Remark 1.16.

We now want to study the properties of $\Phi$ as in (1.3) with the aim of characterizing it. By a Hurwitz-type argument ([BA-DW, 1.5]) the degree of $\Phi$ must be

$$
\operatorname{deg} \Phi=n N .
$$

Let $m_{\Phi}(x, y)$ denote the multiplicity of $\Phi(x)=y$. The relation between the differences of the exponents is

$$
m_{\Phi}(x, y) \Delta_{\mathscr{K}_{N}}(y)=\Delta_{\mathscr{L}_{n}}(x) .
$$

It is clear that to construct $\Phi$ one has to know the "ramification set"

$$
\Gamma=\Phi^{-1}\{0,1, \infty\} \text {. }
$$

The singular points of $\mathscr{L}_{n}$ necessarily belong to $\Gamma$ and if $\gamma \in \Gamma \backslash\left\{e_{1}, e_{2}, e_{3}, \infty\right\}$,

$$
\Delta_{\mathscr{L}}(\gamma)=1 \text {. }
$$

Furthermore

$$
\left(\frac{1}{2}+\frac{1}{2}+\frac{1}{N}\right) n N=\# \Gamma-4+\frac{1}{2}+\frac{1}{2}+\frac{1}{2}+n+\frac{1}{2}, \quad \# \Gamma=n N+2 .
$$

We put $\Gamma=\left\{e_{1}, e_{2}, e_{3}, \infty, \gamma_{1}, \ldots, \gamma_{n N-2}\right\}$. For the possible multiplicities of the elements of $\Gamma$ over $0,1, \infty$ of $\Phi$ (using (1.6), (1.7)) there is Table 1.8. (For a covering $\Phi, m_{\Phi}(x, y)=0$ means that $\left.\Phi(x) \neq y\right)$ :

\section{TABLE 1.8}

\begin{tabular}{c|c|c|c|c|c|c|c|c|c|}
\multicolumn{1}{c}{$e_{1}$} & \multicolumn{1}{c}{$e_{2}$} & \multicolumn{1}{c}{$e_{3}$} & $\infty$ & $\gamma_{1}$ & $\cdots$ & $\gamma_{i}$ & $\cdots$ & $\gamma_{n N-2}$ \\
\cline { 2 - 10 } 0 & 0,1 & 0,1 & 0,1 & $0,2 n+1$ & 0,2 & $\cdots$ & $\cdots$ & $\cdots$ & 0,2 \\
\cline { 2 - 10 } 1 & $0, \frac{N}{2}$ & $0, \frac{N}{2}$ & $0, \frac{N}{2}$ & $0, n N+\frac{N}{2}$ & $0, N$ & $\cdots$ & $\cdots$ & $\cdots$ & $0, N$ \\
\cline { 2 - 10 }$\infty$ & 0,1 & 0,1 & 0,1 & $0,2 n+1$ & 0,2 & $\cdots$ & $\cdots$ & $\cdots$ & 0,2 \\
\hline
\end{tabular}


If $N$ is odd and $n$ is even, there are no new cases (in fact only 1 and 2 occur).

If $N$ is odd and $n$ is odd

4.

\begin{tabular}{|c|c|c|c|c|c|}
\hline & $e_{1}$ & $e_{2}$ & $e_{3}$ & $\infty$ & \\
\hline 0 & 0 & 0 & 1 & 0 & \multirow{3}{*}{$\begin{array}{l}k=\frac{n N-1}{2} \\
p=n \\
\eta=\frac{n N-3-}{2}\end{array}$} \\
\hline & 0 & 0 & 0 & 0 & \\
\hline & 1 & 1 & 0 & $2 n+1$ & \\
\hline
\end{tabular}

$$
(n N \geq 2 n+3)
$$

5.

\begin{tabular}{|c|c|c|c|c|c|}
\hline & $e_{1}$ & $e_{2}$ & $e_{3}$ & $\infty$ & \\
\hline ) & 1 & 1 & 1 & 0 & \multirow{3}{*}{$\begin{aligned} k & =\frac{n N-3}{2} \\
p & =n \\
\eta & =\frac{n N-1-2 n}{2}\end{aligned}$} \\
\hline & 0 & 0 & 0 & 0 & \\
\hline & 0 & 0 & 0 & $2 n+1$ & \\
\hline
\end{tabular}

$$
(n N \geq 2 n+1)
$$

1.10. We indicate by $i \uparrow, i=1,2, \ldots, 5$, the tables obtained from $i, i=$ $1, \ldots, 5$, by interchanging between first and the third lines in each case. We have

Proposition 1.10.1. The cases $i, i \uparrow, i=1, \ldots, 5$, cover all possible multiplicities at the ramification points of a rational map which pulls-back a hypergeometric operator $\mathscr{H}_{N}$ to a Lamé $\mathscr{L}_{n}$.

Proof. The proof of the proposition can be verified directly using the rules (i) and (ii). Note that the cases $i \uparrow, i=1, \ldots, 5$, represent the multiplicities at the ramification points of a covering which is obtained by composition of the automorphism $\frac{1}{x}$ with a covering related to the cases $i, i=1, \ldots, 5$. Q.E.D.

Although the cases $i, i \uparrow, i=1, \ldots, 5$, cover all the possible multiplicities of a covering which transforms by pull-back a hypergeometric operator $\mathscr{H}_{N}$ to a Lamé $\mathscr{L}_{n}$, each case does not uniquely characterize a rational covering map: in fact there exist coverings of $\mathbf{P}^{1}$ by $\mathbf{P}^{1}$ with the same multiplicities at the only ramification points $0,1, \infty$, but not homographic.

1.11. As we said, a Lamé operator with finite dihedral monodromy group of order $2 N$ is a rational pull-back of a hypergeometric one $\mathscr{H}_{N}$ with the same monodromy group [BA], [BA1]. For $N \neq 2$ this map is unique up to homography. But in general a Lamé operator $\mathscr{L}_{n}(n \in \mathbf{N})$ which is a rational pull-back of a hypergeometric $\mathscr{H}_{N}$ will have as monodromy group a dihedral group of order $2 N^{\prime}, N^{\prime} \mid N$ [BA-DW], [BA1].

Thus the problem of "counting" the number, $\mathscr{N} \mathscr{L}(n, N)$, of nonhomographic Lamé operators in $L_{n} \quad\left(n \in \mathbf{N}^{*}\right)$ with given monodromy group dihedral of order $2 N\left(N \in \mathbf{N}^{*}\right)$ is related to the calculation of the number, $\mathscr{N} \mathscr{C}(n, N)$, of all nonhomographic ramified coverings of $\mathbf{P}^{1}$ by $\mathbf{P}^{1}$ (hence rational) which 
pull-back a hypergeometric operator $\mathscr{H}_{N}$ to a Lamé of the type $\mathscr{L}_{n}$. In general we will have

$$
\mathscr{N} \mathscr{L}(n, N) \leq \mathscr{N} \mathscr{C}(n, N)
$$

We will now explain the method for calculating $\mathscr{N} \mathscr{C}(n, N)$ for a given $n$ and $N$. In $\S 2$, we will give the exact formula for the case $n=1$ (2.14) and in the same section, by means of (1.11.1), we also give an exact formula for $\mathscr{N} \mathscr{L}(1, N)(2.15)$.

1.12. We will now see how to calculate such coverings. Consider a rational covering $\Phi$ as in (1.3) which pulls-back a hypergeometric operator $\mathscr{H}_{N}$ to a Lamé $\mathscr{L}_{n}$; it has degree $n N$. Take the fiber over $y \notin\{0,1, \infty\}, \Phi^{-1}(y)$, and enumerate it: $1, \ldots, n N$. By analytic continuation, one obtains a transitive permutation representation of the fundamental group $\Pi\left(\mathbf{P}^{1} \backslash\{0,1, \infty\}, y\right)$ [MA, V],

$$
\Phi_{*}: \Pi\left(\mathbf{P}^{1} \backslash\{0,1, \infty\}, y\right) \rightarrow \sigma_{n N} ;
$$

the image subgroup of this representation will be called the monodromy covering group. The fundamental group $\Pi\left(\mathbf{P}^{1} \backslash\{0,1, \infty\}, y\right)$ is generated by the paths $\tau, \rho, \sigma$ around $0,1, \infty$ (resp.) with relation $\tau \rho \sigma=1$. The cycle decomposition of the permutations $\Phi_{*}(\tau), \Phi_{*}(\rho), \Phi_{*}(\sigma)$ represents the multiplicities of the points above $0,1, \infty$ (respectively). In our cases the multiplicities of the ramification points are given by the various $\mathscr{C}(n, N)$ cases.

On the other hand we have a one-to-one correspondence between the unramified coverings of $\mathbf{P}^{1} \backslash\{0,1, \infty\}$ of degree $n N$ and the transitive representations of the fundamental group of $\mathbf{P}^{1} \backslash\{0,1, \infty\}$ in the symmetric group of order $n N, \sigma_{n N}$.

In fact we know from elementary algebraic topology that all spaces are assumed arcwise connected and locally arcwise connected.

Theorem 1.12.2 (cf. [MA, V.4.2]). Let $X$ be a topological space and $p: X^{\circ} \rightarrow X$ be an unramified covering. Consider $x_{0}$ a point of $X$. Then the subgroups $p_{*} \Pi\left(X^{\circ}, \tilde{x}\right)$ where $\tilde{x} \in p^{-1}\left(x_{0}\right)$ form a whole conjugacy class of subgroups of $\Pi\left(X, x_{0}\right)$.

Again from [MA, V.10.1] we have this constructive result.

Theorem 1.12.3. Let $X$ be a topological space which admits a universal covering. Then for each conjugacy class of subgroups of $\Pi(X, x), x \in X$, there exists an unramified covering $\left(X^{\circ}, p\right)$ of $X$ such that $p_{*} \Pi\left(X^{\circ}, \tilde{x}\right), \tilde{x} \in p^{-1}(x)$, belongs to the given conjugacy class.

On the other hand a conjugacy class of subgroups is characterized uniquely by the associated covering.

Theorem 1.12.4 [MA, V.6.6]. Two covering spaces $\left(X_{1}, p_{1}\right),\left(X_{2}, p_{2}\right)$ of $X$ are isomorphic if and only if there exist two points $x_{1} \in X_{1}$ and $x_{2} \in X_{2}$ such that $p_{1}\left(x_{1}\right)=p_{2}\left(x_{2}\right)=x_{0} \in X$ and the subgroups $p_{1 *} \Pi\left(X_{1}, x_{1}\right)$ and $p_{2 *} \Pi\left(X_{2}, x_{2}\right)$ belong to the same conjugacy class. 
$\mathbf{P}^{1} \backslash\{0,1, \infty\}$ is a Riemann surface: it admits a universal covering. Consider a transitive representation of $\Pi\left(\mathbf{P}^{1} \backslash\{0,1, \infty\}, y\right)$ on $\sigma_{n N}$ connected with a case $\kappa \in \mathscr{C}(n, N)$; i.e. it will have a cycles decomposition for the permutations $\Phi_{*}(\tau), \Phi_{*}(\rho)$ and $\Phi_{*}(\sigma)$ given by the lines in the table $\kappa$. Such a representation characterizes a whole conjugacy class of subgroups of $\sigma_{n N}$ [SE-TR, pp. 207-210]: hence a topological unramified covering $T^{\prime}$ of $\mathbf{P}^{1} \backslash\{0,1, \infty\}$ of degree $n N$

$$
p: T^{\prime} \rightarrow \mathbf{P}^{1} \backslash\{0,1, \infty\}
$$

where $p$ is a local homeomorphism and a proper map. The representation related to $p$ as in (1.1) has image in $\sigma_{n N}$ which is a subgroup conjugate to that from which we started. By the fact that $p$ is a local homeomorphism, $T^{\prime}$ itself is a Riemann surface. We can now apply the following:

Theorem 1.12.5 [FO, Theorems 8.4 and 8.5]. Let $X$ be a Riemann surface, and $A$ be a discrete closed subset. Let $X^{\prime}=X \backslash A$ and indicate by $p: Y^{\prime} \rightarrow X^{\prime}$ a proper unramified covering map, with $Y^{\prime}$ a Riemann surface. Then $p$ extends to a ramified proper covering $\Phi: Y \rightarrow X$, with $Y$ a Riemann surface, such that $Y \backslash \Phi^{-1}\left(X^{\prime}\right)$ is biholomorphic via a fiber-preserving map to $Y^{\prime}$. The Riemann surface $Y$ is unique up to covering transformations.

So we can "complete uniquely" our covering $T^{\prime}$ to a covering $T$ over $\mathbf{P}^{1}$. The multiplicities over the branched points (which in our case are $0,1, \infty$ ) are characterized by the local monodromy, hence by the cycle decompositions of the permutations which represent the paths around the branched points. In our case, for every representation (up to conjugation) of $\Pi\left(\mathbf{P}^{1} \backslash\{0,1, \infty\}, y\right)$ in $\sigma_{n N}$ related to $\kappa \in \mathscr{C}(n, N)$ we obtain a ramified (only over $\left.0,1, \infty\right)$ proper covering of $\mathbf{P}^{1}$ of degree $n N$. By a Hurwitz-type argument this covering space necessarily is $\mathbf{P}^{1}$.

1.13. In this sense, if for given $n \in \mathbf{N}^{*}$ and $N \in \mathbf{N}^{*}$ and for each case $\kappa$ in $\mathscr{C}(n, N)$ (compatible of course with the choice of $n, N)$ we can calculate all the transitive subgroups of $\sigma_{n N}$ (up to conjugation) with the cycle decomposition and structure deriving from $\kappa$ as above (i.e. a subgroup generated by two permutations $\Phi_{*}(\tau)$ and $\Phi_{*}(\rho)$ with cycle decomposition given by (resp.) the first two lines of $\kappa$ and such that the composition $\Phi_{*}(\tau) \circ \Phi_{*}(\rho)$ has cycle decomposition as the third line in $\kappa$ ), we actually calculate the number of nonhomographic coverings of $\mathbf{P}^{1}$ by $\mathbf{P}^{\mathbf{1}}$ with prescribed ramifications over 0 , $1, \infty$. Hence we will give the number $\mathscr{N} \mathscr{C}(n, N)$ of all inequivalent (i.e. nonhomographic, because the covering space is $\mathbf{P}^{1}$ ) coverings which pull-back a hypergeometric operator $\mathscr{H}_{N}$ into a Lamé $\mathscr{L}_{n}$.

1.14. Finally, the problem is to find for each case $\kappa$ in $\mathscr{C}(n, N)$ all the subgroups of $\sigma_{n N}$ (up to conjugation) generated by $\Phi_{*}(\tau)$ and $\Phi_{*}(\rho)$ (for which we know only the cycle decomposition: first and second row in case $\kappa$ ) such that

$$
\Phi_{*}(\tau) \circ \Phi_{*}(\rho)
$$

has a cycle decomposition exactly equal to $\Phi_{*}(\sigma)$ (third line in the case $\left.\kappa\right)$ : in fact $\Phi_{*}(\tau) \circ \Phi_{*}(\rho)=\Phi_{*}(\sigma)^{-1}$ has the same cycle decomposition as $\Phi_{*}(\sigma)$. For each $\kappa$ we denote by $\mathscr{N}(\kappa)$ the number of such conjugacy classes of subgroups. $\mathscr{N}(\kappa)$ will also be the number of nonhomographic coverings of $\mathbf{P}^{1}$ by $\mathbf{P}^{1}$ with exactly the multiplicities over the ramification points given by $\kappa$. 
Observation 1.15. The Lamé operators related to the pull-backs in the case $i$ and $i \uparrow$ (for each $i=1,2, \ldots, 5$ ) are the same. In fact as we stated in the proof of 1.10.1 the cases $i$ and $i \uparrow$ are connected by an inversion $\frac{1}{x}$ in the target. This is equivalent to taking first the pull-back of a hypergeometric operator $\mathscr{H}_{N}$ by $\frac{1}{x}$ and afterwards applying one of the pull-backs related to $i$. But the operator $\mathscr{H}_{N}=L_{\frac{1}{2}, \frac{1}{N}, \frac{1}{2}}$ after the $\frac{1}{x}$ transformation maintains the same projective monodromy and normal form (1.4). It follows that no new operators are constructed by $i \uparrow$.

So, for our computation, it is enough to study the cases $i, i=1,2, \ldots, 5$; and from now on we will denote by $\mathscr{C}(n, N)$ only these cases.

Remark 1.16. We want to make some comments about the notion of equivalence up to homography in $L_{n}$. As one can easily see, only homographies with $\infty$ as a fixed point operate in the set $L_{n}$ (of course in the independent variable $x)$. This is consistent with our constructions. In fact, a homography $\chi$ which makes two coverings isomorphic

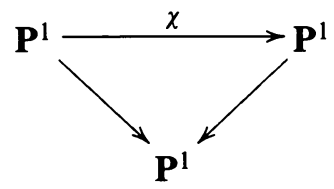

has to respect the fibers and the multiplicities. The ramifications and the multiplicities for the coverings related to rational functions which pull-back a hypergeometric operator $\mathscr{H}_{N}$ into a Lamé $\mathscr{L}_{n}$ are ruled by the previous cases $i$ and $i \uparrow$. In each case $\infty$ has an exceptional multiplicity in its fiber, so $\chi$ has to send $\infty$ to itself. Actually, throughout our article we could restrict to homographies of the type $a x+b$.

\section{2}

In this section we will give an explicit calculation for the case $n=1$. Namely, in the first part of this section we will deal with the calculation of all the coverings which pull back a hypergeometric operator $\mathscr{H}_{N}$ into a Lamé of type $\mathscr{L}_{1}$. In the second part, we will obtain information about the number of inequivalent Lamé operators, $\mathscr{L}_{1}$, with finite dihedral monodromy group of order $2 N$.

2.1. By $\S 1$, the problem of calculating the coverings which pull back a hypergeometric operator $\mathscr{H}_{N}$ into a Lamé of type $\mathscr{L}_{1}$ is equivalent to calculating the number of nonhomographic ramified coverings

$$
\Phi: \mathbf{P}^{1} \rightarrow \mathbf{P}^{1}
$$

of degree $N$, ramified over $0,1, \infty$ with cycle decomposition for the monodromy covering group given by the cases $\mathscr{C}(1, N)$ and this turns out to be equal to the number of all nonconjugate subgroups of $\sigma_{N}$ with the structure related to $\mathscr{C}(1, N)$ as in $1.12,1.13$ and 1.14. In fact for each case $\kappa \in \mathscr{C}(1, N)$ we will need to characterize all the subgroups of $\sigma_{N}$ generated by two permutations (which we will indicate (resp.) $\Phi_{*}(\tau)$ and $\Phi_{*}(\rho)$ ) which have the cycle decomposition given (resp.) by the first two lines in the case $\kappa$ and such that the composition of these two permutations $\left(\Phi_{*}(\tau) \circ \Phi_{*}(\rho)=\Phi_{*}(\sigma)^{-1}\right)$ has exactly the same cycle decomposition as the third line in $\kappa(1.14)$. 
We will indicate with the same symbols $\Phi_{*}(\rho), \Phi_{*}(\tau)$ and $\Phi_{*}(\sigma)$ also their cycle decompositions. In general $\Phi_{*}(\rho)$ consists of a large cycle of length $N$ or, in case 3 only, two cycles of length $\frac{N}{2}$, with $N$ even; $\Phi_{*}(\tau)$ consists of cycles of length 2 or 1 . The composition of the two must have the same cycle decomposition as $\Phi_{*}(\sigma)$ (third line of $\kappa$ ) and so it may have cycles of length 2 and 1, but in every case one and only one cycle of length 3. (Notation: a $q$-cycle for $q \in \mathbf{N}^{*}$ is a cycle of length $q$.)

2.2. To characterize such subgroups (up to conjugation) we indicate $\Phi_{*}(\rho)$ by a diagram of the type:

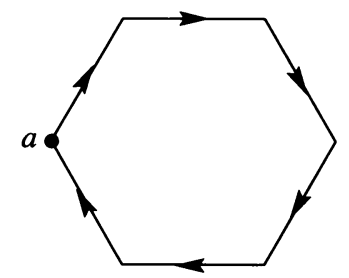

(if $a$ is a vertex $\Phi_{*}(\rho)(a)$ is the target of the arrow starting in $a$ ) and by a dashed line - - - - - the 2-cycle and by $/ /$ the 1-cycle in $\Phi_{*}(\tau)$; for the composition of the two $\left(\Phi_{*}(\tau) \circ \Phi_{*}(\rho)\right)$ we may use the diagram:

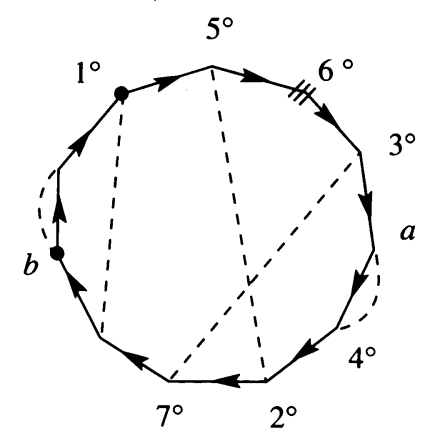

Remark. We always assume that in a convex diagram as in (2.3) the 2-cycles of $\Phi_{*}(\tau)$ (dashed lines) are drawn in the interior, except in the case in which they are between two vertices connected by a side of $\Phi_{*}(\rho)$ (this situation will be denoted by $(\dagger)$ in Remark 2.3.2). Note that (2.3) does not necessarily correspond to a case that actually occurs in $\mathscr{C}(1, N)$.

2.3.1. In order to find the cycles of the composition (which in our situation must be those of $\Phi_{*}$; see 1.14) one starts from a vertex (say $1^{\circ}$ in (2.3)) and applies $\Phi_{*}(\rho)$ and in the target one applies $\Phi_{*}(\tau)$ : the result, $2^{\circ}$, is where $1^{\circ}$ is sent by $\Phi_{*}(\tau) \circ \Phi_{*}(\rho)$. In the following we will denote such a situation by $1^{\circ} \hookrightarrow 2^{\circ}$. In order to find the length of the cycle one goes on until one comes back to $1^{\circ}$.

Remark 2.3.2. In (2.3) there is a 7-cycle for the composition. Only a situation of the type:

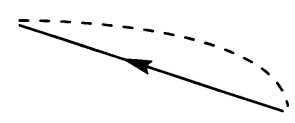


gives a 1-cycle in the composition ( $a, b$ in (2.3)). In conclusion, the composition in (2.3) has a cycle of length 7 , two 1-cycles and one cycle of length 2 (so it will not be a diagram of a case in $\mathscr{C}(1, N)$ ).

2.3.3. We recall that for every case in $\mathscr{C}(1, N)$ (cf. 2.1 ) there must be exactly one 3-cycle in the composition. A diagram of the type just introduced (2.3) characterizes a subgroup of the symmetric group up to conjugation. So two diagrams must be considered the same if one comes from the other by a rotation. In the sequel we identify a diagram with a subgroup up to conjugation. We give now some information about the structure of the previous diagrams using the cycle decomposition.

Proposition 2.4. Case 3 never occurs.

Proof. In this case $\Phi_{*}(\rho)$ is made up to two "large" cycles of length $\frac{N}{2}$. We call $A$ the one which contains the unique $(\dagger)$ diagram. (In fact the composition has only one cycle of length 1 , i.e. a fixed point.) The other will be denoted by $B$. The permutation group represents the monodromy group of a covering and thus it has to be connected (transitive). So, there exists at least one 2-cycle of $\Phi_{*}(\tau)$ connecting the two $\frac{N}{2}$-cycles (from now on, by connecting we mean a cycle which connects the two large $\frac{N}{2}$-cycles). In the sense of the rotation of $A$ we fix the first connecting 2-cycle of $\Phi_{*}(\tau), \alpha$, which follows the ( $\dagger$ ) diagram. We obtain the following:
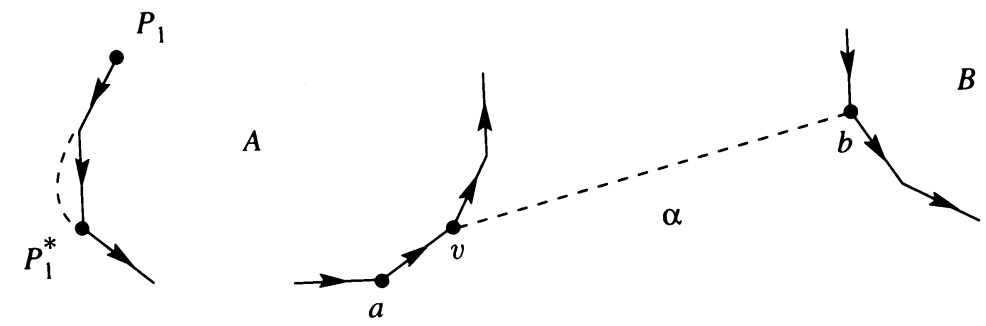

We recall that the permutation given by the composition $\Phi_{*}(\tau) \circ \Phi_{*}(\rho)$ must have only one 3-cycle. Using the diagram (2.5) we have $a \hookrightarrow b(2.3 .1)$ for the composition and necessarily $a$ and $b$ belong to a 3-cycle: we cannot close $a$ and $b$ in a 2-cycle in the composition because if this were the case then $b \hookrightarrow a$ and this fact would contradict the hypothesis on $\alpha$. Consider then as in (2.5) $P_{1} \hookrightarrow P_{1}^{*}$ in $A$. If $P_{1}$ and $P_{1}^{*}$ belong to a 2 -cycle necessarily $P_{1}^{*} \hookrightarrow P_{1}$ and the diagram will be

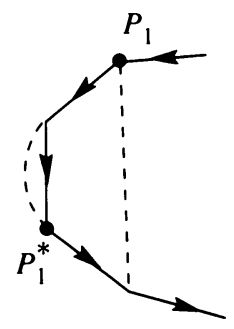

We may continue in this way in $A$ until we find $P_{j} \hookrightarrow P_{j}^{*} \quad(j \in \mathbf{N}, j \geq 1)$, but $P_{j}^{*} \leftrightarrow P_{j}$ in the composition (this necessarily happens because at worst 
we reach $v$ ). Hence $P_{j}^{*}$ and $P_{j}$ are in the only 3-cycle of the composition. We then have that $a, b, P_{j}, P_{j}^{*}$ are all elements of a 3-cycle: two of them (at least) have to coincide. There are only two possible cases: either $a \equiv P_{j}$ or $a \equiv P_{j}^{*}$. In the first case, necessarily $j>1$, and we would also have $P_{j-1}=v$ : but this is impossible because a connecting 2-cycle starts from $v$ while by construction $P_{j-1} \hookrightarrow P_{j-1}^{*}$ in $A$. In the second case, i.e. $a \equiv P_{j}^{*}$, the 3 -cycle of the composition consists of $a \equiv P_{j}^{*}, b, P_{j}$. We know that $a \hookrightarrow b$, $P_{j} \hookrightarrow P_{j}^{*} \equiv a$, so necessarily we would have $b \hookrightarrow P_{j}$. The diagram is as follows:
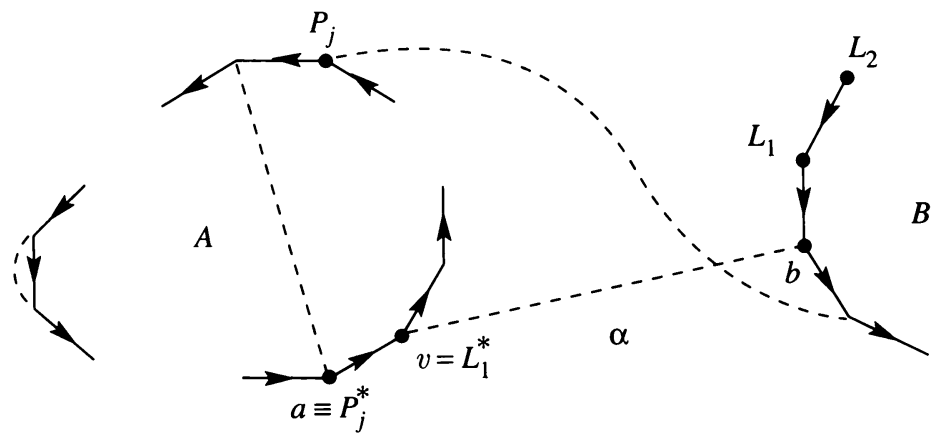

From (2.5.1) we have $L_{1} \hookrightarrow v=L_{1}^{*}$. But the unique 3-cycle of the composition has been constructed before; hence $v=L_{1}^{*} \hookrightarrow L_{1}$. We may continue in this way until one of the following 3 cases occurs:

(i) $L_{k}^{*}=P_{j} \quad(k \in \mathbf{N}, k>1)$.

(ii) From $L_{k}\left(L_{k} \hookrightarrow L_{k}^{*}\right)$ we have a 2-cycle of $\Phi_{*}(\tau)$ connecting two vertices of $B \quad(k \in \mathbf{N}, k \geq 1)$.

(iii) From $L_{k}$ we have a connecting 2-cycle of $\Phi_{*}(\tau)$, but it does not allow $L_{k}^{*} \hookrightarrow L_{k} \quad(k \in \mathbf{N}, k \geq 1)$.

Consider the case (i), i.e. $L_{k}^{*}=P_{j}$. In this case $L_{k} \hookrightarrow L_{k}^{*}=P_{j}$ and $L_{k}^{*}=$ $P_{j} \hookrightarrow a$ : but since the composition has to have just one 3-cycle, we are forced to have $b=L_{k}$. But this is impossible: in fact $b=L_{k}$ means that we have marked all the $\frac{N}{2}$ vertices of $B$ by $L_{1}, \ldots, L_{k}$, while the corresponding $L_{1}^{*}, \ldots, L_{k}^{*}$ may just cover the vertices between $a$ and $P_{j}$ (hence in number smaller or equal to $\frac{N}{2}-2$ ). The cases (ii) and (iii) are not admissible either (of course we suppose that the $L_{k}^{*}$ 's are in the $A$ diagram between $v$ and $P_{j}$ ): in fact we will have another 3 -cycle different from the previous $a, b, P_{j}$ while 3 has only one 3-cycle in the composition. So all the cases presented by the diagram (2.5.1) fail to accomplish the requests of case 3 : it is impossible to have case 3. Q.E.D.

Proposition 2.6. In every case $\kappa$ of $\mathscr{C}(1, N)$ it is impossible to have two crossing 2-cycles of $\boldsymbol{\Phi}_{*}(\tau)$.

Proof. In view of (2.4), we need only study the cases in which $\Phi_{*}(\rho)$ consists of a single cycle of order $N$. In all these cases there exists either a (†) diagram or a 1-cycle of $\Phi_{*}(\tau)$ : we fix one of those and we denote it by $\mathscr{F}$. After $\mathscr{F}$, in the sense of rotation, $\Phi_{*}(\rho)$ we consider the first 2-cycle of $\Phi_{*}(\tau), \alpha$, crossed. On the other hand we also fix the first vertex after $\alpha, L$, from which we have 
a 2-cycle of $\Phi_{*}(\tau)$ crossing $\alpha$. We will have the diagram

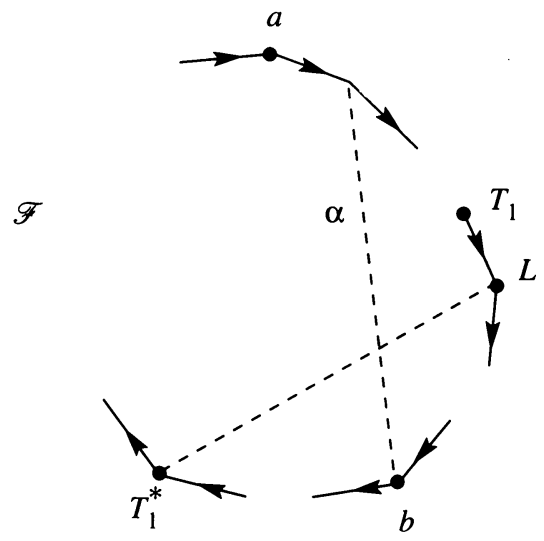

By the diagram we will have $a \hookrightarrow b$ and $a, b$ are not in a 2-cycle of the composition: in fact, in order to have a 2-cycle of the composition we should have $b \hookrightarrow a$ and we would have a diagram of the type:

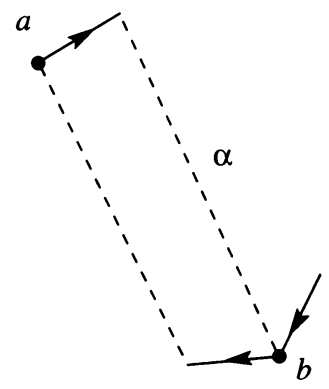

and this contradicts the hypothesis on $\alpha$. So $a$ and $b$ belong to the unique 3-cycle of the composition. In the diagram (2.6.1) we also consider $T_{1} \hookrightarrow$ $T_{1}^{*}$ : by our hypothesis on $L, T_{1}$ and $T_{1}^{*}$ belong to the unique 3-cycle of the composition. Hence in order to have a unique 3-cycle at least two of $a, b, T_{1}$, $T_{1}^{*}$ must coincide. But this is impossible without contradicting our hypotheses: in fact by construction $T_{1} \not \equiv T_{1}^{*}, b \not \equiv a, a \not \equiv T_{1}$ and $b \not \equiv T_{1}^{*}$. If $a \equiv T_{1}^{*}$, then $\alpha$ will not be the first 2-cycle crossed. Also the case $b \equiv T_{1}$ does not happen. Hence $a, b, T_{1}, T_{1}^{*}$ must all be different and so we cannot have just one 3-cycle. Thus crossing cycles for $\Phi_{*}(\tau)$ are not allowed in the construction of the subgroups of $\sigma_{N}$ having cycle decompositions and structures as in the case $\mathscr{C}(1, N)$. Q.E.D.

Remark 2.7. A change in the orientation of $\Phi_{*}(\rho)$ does not invalidate the previous proofs.

After these general propositions about the structure of the groups, we calculate now the number of these groups. We start with the case $N$ even. In this hypothesis in $\mathscr{C}(1, N)$ there are only 1 and 2 (cf. 2.4 ). 
Proposition 2.8. If $N$ is even, the number of nonconjugate groups in case 1 is $(N \geq 6)$

$$
\begin{gathered}
\frac{N}{6} \cdot \frac{N-6}{4}+1 \quad \text { if } N \equiv 0 \bmod 3, \\
\frac{N-2}{6} \cdot \frac{N-4}{4} \quad \text { if } N \equiv 1,2 \bmod 3 .
\end{gathered}
$$

Proof. In this case the composition $\Phi_{*}(\sigma)$ has three 1-cycles and one 3-cycle. It follows that the diagram (of the type 2.3) for case 2 will have three $(\dagger)$ pictures. The problem is to distribute the 2-cycles of $\Phi_{*}(\tau)$ (it has only 2cycles in this case) without crossing and three $(\dagger)$. The generic picture will be

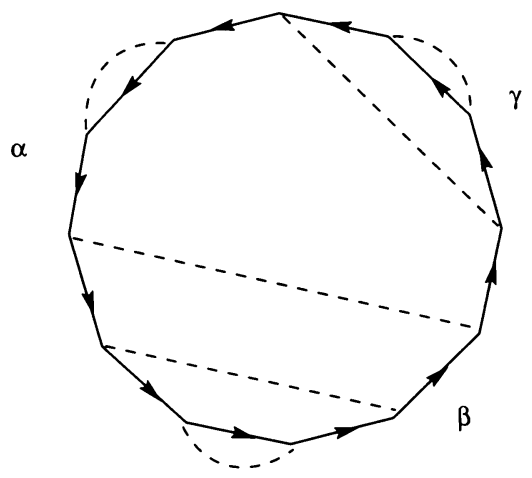

where we denote by $\alpha \quad \beta, \gamma$ the number of 2-cycles of $\Phi_{*}(\tau)$ "in that side" (excluding the one involved in (†)). Of course we can have one or more of $\alpha$, $\beta, \gamma$ equal to zero (for example $\alpha$ is equal to 0 in the previous picture). In this case in that side (where one of the $\alpha, \beta, \gamma$ is zero), we must have only the ( $\dagger$ )-diagram. (Note that one is forced to construct the previous picture: in fact we need just one 3-cycle then 2 and 1-cycles and not crossing cycles.)

We must have:

$$
\alpha+\beta+\gamma=\frac{N}{2}-3, \quad \alpha, \beta, \gamma \geq 0 .
$$

In order to characterize all the groups with the data $\alpha, \beta, \gamma$ and to avoid symmetries we study the triples: $\gamma \geq \beta \geq \alpha$ for a fixed orientation of $\Phi_{*}(\rho)$ : the opposite diagram (i.e. the same diagram but changing the sense of rotation (orientation) of $\Phi_{*}(\rho)$ ) will give for each choice of $\alpha \leq \beta \leq \gamma$ another not conjugate group. This will not be the case if at least two of $\alpha, \beta, \gamma$ are equal. So denoting $P=\frac{N}{2}-3$ and $a=\left[\frac{P}{3}\right]$, the number of total possibilities is

$$
2 \sum_{\alpha=0}^{a} \sum_{\beta=\alpha}^{\left[\frac{P-\alpha}{2}\right]} 1-((a+1)+\#\{\text { even in }[P-a, P]\}-\varepsilon)
$$

where $\varepsilon=1$ if $P \equiv 0 \bmod 3$, zero otherwise $((a+1)$ is linked with $\alpha=\beta$, while the other term to $\beta=\gamma$. The case $\alpha=\beta=\gamma$, if possible $(P \equiv 0 \bmod 3)$, is counted in this way twice; the multiplication by 2 is related to the change of sense of $\left.\Phi_{*}(\rho)\right)$. 


$$
\begin{aligned}
= & 2 \sum_{\alpha=0}^{a}\left(\left[\frac{P-\alpha}{2}\right]-\alpha+1\right)-((a+1)+\#\{\text { even in }[P-a, P]\}-\varepsilon) \\
= & 2\left(\sum_{\alpha=0}^{a}\left(\frac{P}{2}-\frac{3 \alpha}{2}+1\right)-\frac{\#\{\text { odd in }[P-a, P]\}}{2}\right) \\
& -((a+1)+\#\{\text { even in }[P-a, P]\}-\varepsilon) \\
= & \sum_{\alpha=0}^{a}(P-3 \alpha+2)-2(a+1)+\varepsilon \\
= & P(a+1)-\frac{3}{2} a(a+1)+2(a+1)-2(a+1)+\varepsilon \\
= & P(a+1)-\frac{3}{2} a(a+1)+\varepsilon .
\end{aligned}
$$

For the various reductions of $P \bmod 3$ we obtain

(i) if $P \equiv 0 \bmod 3, a=\frac{P}{3}$,

$$
\frac{P+3}{3} \cdot \frac{P}{2}+1
$$

(ii) If $P \equiv 1 \bmod 3, a=\frac{P-1}{3}$, if $P \equiv 2 \bmod 3, a=\frac{P-2}{3}$,

$$
\frac{P+1}{3} \cdot \frac{P+2}{2} \text {. }
$$

We put $P=\frac{N}{2}-3$, and by substitution we get the proposition. Q.E.D.

Proposition 2.9. If $N$ is even, the number of nonconjugate subgroups in case 2 is

$$
\frac{N-2}{2} \cdot \frac{N}{4}, \quad N \geq 4
$$

Proof. Now the composition (i.e. the third line in the 2-case) has only one 1 -cycle so we will have only one $(t)$-diagram. This $(t)$-diagram and the two 1-cycles of $\Phi_{*}(\tau)$ have to be distributed as in the following diagram (see the first line in the 2-case):

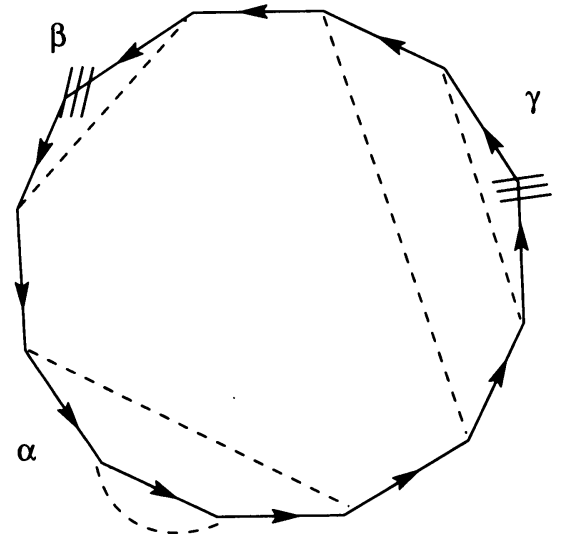


With the notation of proof of 2.8 :

$$
\alpha+\beta+\gamma=\frac{N}{2}-2, \quad \alpha, \beta, \gamma \geq 0 .
$$

If we denote by $\alpha$ the number of 2-cycles of $\Phi_{*}(\tau)$ "in the same side" of the ( $\dagger$ )-diagram, the only type of symmetry we have to worry about when we consider the opposite diagram for $\Phi_{*}(\rho)$ is the case $\beta=\gamma$. So, if we put $\beta \leq \gamma$, the total number of nonconjugate subgroups is:

$$
\begin{aligned}
2 \sum_{\alpha=0}^{\frac{N}{2}-2}\left[\frac{N}{2} \sum_{\beta=0}^{\frac{N}{2}-\alpha}\right] & 1-\#\left\{\text { even in }\left[0, \frac{N}{2}-2\right]\right\} \\
= & 2\left(\sum_{\alpha=0}^{\frac{N}{2}-2}\left(\frac{N}{4}-1-\frac{\alpha}{2}+1\right)-\frac{\left.\# \text { odd in }\left[0, \frac{N}{2}-2\right]\right\}}{2}\right) \\
& -\#\left\{\text { even in }\left[0, \frac{N}{2}-2\right]\right\} \\
= & \frac{N}{2}\left(\frac{N}{2}-2+1\right)-\frac{1}{2}\left(\frac{N}{2}-2\right)\left(\frac{N}{2}-2+1\right)-\left(\frac{N}{2}-2+1\right) \\
= & \left(\frac{N}{2}-1\right)\left(\frac{N}{2}-\frac{N-4}{4}-1\right)=\frac{N-2}{2} \cdot \frac{N}{4} . \text { Q.E.D. }
\end{aligned}
$$

Remark 2.10. In the case $N$ even $(n=1)$, the total number of subgroups for $N \geq 4$ is:

$$
\begin{gathered}
\left(\frac{N}{6} \frac{N-6}{4}+1+\frac{N-2}{2} \frac{N}{4}\right)=\frac{1}{6}(N-1)(N-2)+\frac{2}{3} \quad \text { if } N \equiv 0 \bmod 3, \\
\left(\frac{N-2}{6} \frac{N-4}{4}+\frac{N-2}{2} \frac{N}{4}\right)=\frac{1}{6}(N-1)(N-2) \quad \text { if } N \equiv 1,2 \bmod 3 .
\end{gathered}
$$

We have to compute now the case $N$ odd. Here we have only cases 4 and 5 (see Remark 2.1).

Proposition 2.11. If $N$ is odd, the number of nonconjugate subgroups in case 4 is

$$
\frac{N-3}{2} \cdot \frac{N-1}{4}, \quad N \geq 5 .
$$

Proof. In this case 4, the composition of the first two lines has two 1-cycles: so two ( $\dagger$ )-pictures appear in the diagram related to this case. We have to distribute these two pictures and the only 1-cycle of $\Phi_{*}(\tau)$. In this situation (as in case 2), if we relate $\alpha$ to the 1-cycle of $\Phi_{*}(\tau)$ we have

$$
\alpha+\beta+\gamma=\frac{N-5}{2}, \quad \alpha, \beta, \gamma \geq 0,
$$


and the subgroups are

$$
\begin{aligned}
2 \sum_{\alpha=0}^{\frac{N-5}{2}}\left[\frac{\frac{N-5}{2}-\alpha}{2}\right] & \sum_{\beta=0}^{2} 1-\#\left\{\text { even in }\left[0, \frac{N-5}{2}\right]\right\} \\
= & 2\left(\sum_{\alpha=0}^{\frac{N-5}{2}}\left(\frac{N-5}{4}-\frac{\alpha}{2}+1\right)-\frac{\#\left\{\text { odd in }\left[0, \frac{N-5}{2}\right]\right\}}{2}\right) \\
& -\#\left\{\text { even in }\left[0, \frac{N-5}{2}\right]\right\} \\
= & \frac{N-5}{2}\left(\frac{N-5}{2}+1\right)-\frac{1}{2} \frac{N-5}{2}\left(\frac{N-5}{2}+1\right) \\
& +2\left(\frac{N-5}{2}+1\right)-\left(\frac{N-5}{2}+1\right) \\
= & \frac{N-3}{2} \frac{N-1}{4} \text {. Q.E.D. }
\end{aligned}
$$

Proposition 2.12. If $N$ is odd, the number of nonconjugate subgroups in case 5 for $N \geq 3$ is

$$
\begin{aligned}
& \frac{N+3}{6} \cdot \frac{N-3}{4}+1 \text { if } N \equiv 0 \bmod 3 \\
& \frac{N+1}{6} \cdot \frac{N-1}{4} \text { if } N \equiv 1,2 \bmod 3 .
\end{aligned}
$$

Proof. Here we have to distribute three 1-cycles of $\Phi_{*}(\tau)$. The symmetries will be as in Proposition 2.8, but with

$$
\alpha+\beta+\gamma=\frac{N-3}{2}, \quad \alpha, \beta, \gamma \geq 0 \text {. Q.E.D. }
$$

Remark 2.13. For $N$ odd $(N \geq 3)$, the total number of nonconjugate subgroups is

$$
\begin{gathered}
\left(\frac{N+3}{6} \frac{N-3}{4}+1+\frac{N-3}{2} \frac{N-1}{4}\right)=\frac{1}{6}(N-1)(N-2)+\frac{2}{3} \quad \text { if } N \equiv 0 \bmod 3, \\
\left(\frac{N+1}{6} \frac{N-1}{4}+\frac{N-3}{2} \frac{N-1}{4}\right)=\frac{1}{6}(N-1)(N-2) \quad \text { if } N \equiv 1,2 \bmod 3 .
\end{gathered}
$$

Each class of subgroups (up to conjugation) corresponds to the monodromy of a covering of $\mathbf{P}^{1}$ by $\mathbf{P}^{1}$; hence it is associated to a rational map

$$
\Phi: \mathbf{P}^{1} \rightarrow \mathbf{P}^{1}
$$

which transforms by pull-back a hypergeometric operator $\mathscr{H}_{N}$ into a Lamé of the type $\mathscr{L}_{1}$. So we can state

Theorem 2.14. The number $\mathscr{N} \mathscr{C}(1, N)$, of nonhomographic coverings of $\Phi$ : $\mathbf{P}^{1} \rightarrow \mathbf{P}^{1}$ which transform by pull-back a hypergeometric operator $\mathscr{H}_{N}$ into a 
Lamé of the type $\mathscr{L}_{1}$ is exactly

$$
\begin{gathered}
\frac{1}{6}(N-1)(N-2)+\frac{2}{3} \quad \text { if } N \equiv 0 \bmod 3, \\
\frac{1}{6}(N-1)(N-2) \quad \text { if } N \equiv 1,2 \bmod 3 . \text { Q.E.D. }
\end{gathered}
$$

In particular we obtain

Corollary 2.14.1. A Lamé operator of the type $\mathscr{L}_{1}$ never has monodromy group dihedral of order 4 .

Proof. In fact such a Lamé operator would be a rational pull-back of a hypergeometric one of the type $\mathscr{H}_{2}, N=2$. Q.E.D.

Remark 2.14.2. This result was already stated by Baldassarri in [BAT]. Corollary 2.14.1 is also true not only for $n=1$ but for a general $n$ [CO].

In this second part we use Theorem 2.14 in order to give an answer to our problem of calculating the number, $\mathscr{N} \mathscr{L}(1, N)$, of inequivalent Lamé operators of the type $L_{1}$ with finite dihedral monodromy group of order $2 N$ for $N \in$ $\mathbf{N}^{*}$. We will carry out such a calculation using our knowledge of the number $\mathscr{N C}(1, N)(2.14)$ of nonhomographic covering maps of $\mathbf{P}^{1}$ by $\mathbf{P}^{1}$ which pullback a hypergeometric operator $\mathscr{H}_{N}$ into a Lamé $\mathscr{L}_{1}$. In fact we have the following general statement.

Theorem 2.15. In the previous notation, let $\mathscr{N} \mathscr{L}(1, N)$ be the number of inequivalent Lamé operators $\mathscr{L}_{1}$ which have finite dihedral monodromy group of order $2 N, N \in \mathbf{N}^{*}, N \neq 2$, and let $\mathscr{N} \mathscr{C}(1, N)$ be the number of nonhomographic (rational) coverings of $\mathbf{P}^{1}$ by $\mathbf{P}^{1}$ which transform by pull-back a hypergeometric $\mathscr{H}_{N}$ into a Lamé $\mathscr{L}_{1}$. We have

$$
\mathscr{N} \mathscr{C}(1, N)=\sum_{N^{\prime} \mid N, N^{\prime} \neq 2} \mathscr{N} \mathscr{L}\left(1, N^{\prime}\right) .
$$

Proof. The fact that $N^{\prime} \neq 2$ follows from 2.14.1. In general an operator which is a rational pull-back of the hypergeometric $\mathscr{H}_{N}$ always has a finite monodromy group which is a subgroup of the dihedral group of order $2 N$. Thus we need only to prove that every Lamé operator $\overline{\mathscr{L}}$ with finite monodromy group of order $2 N^{\prime}$ with $N^{\prime} \mid N, N^{\prime} \neq 2$ may be constructed as a rational pull-back of the hypergeometric operator $\mathscr{H}_{N}$.

For a Lamé operator $\overline{\mathscr{L}}$ as before, we actually know the existence of a rational map (unique, because $N \neq 2[\mathrm{BA}]$ ) which presents $\overline{\mathscr{L}}$ as a rational pull-back of the hypergeometric operator $\mathscr{Z}_{N^{\prime}}$. We just need to show the existence (and unicity up to homography) of a rational map $\Phi$ which transforms by pull-back the hypergeometric operator $\mathscr{H}_{N}$ into $\mathscr{H}_{N^{\prime}}$. By the Hurwitz formula (1.5.1) (cf. [BA-DW, 1.5]) the degree of $\Phi$ is

$$
\operatorname{deg} \Phi=N / N^{\prime} .
$$

We may study $\Phi$ exactly as in $\S 1$ : the only ramification points are over 0,1 , $\infty$. The number of points in the fibers over $0,1, \infty$ is $N / N^{\prime}+2$; among those there will be $0,1, \infty$ because they are singular points of $\mathscr{H}_{N^{\prime}}$. We indicate the others (which are not singular points for $\mathscr{H}_{N^{\prime}}$ ) by $\gamma_{1}, \ldots, \gamma_{N / N^{\prime}-1}$. In this case Table 1.8 becomes 


\begin{tabular}{r|c|c|c|c|c|c|c|c|}
\multicolumn{2}{c}{0} & \multicolumn{1}{c}{1} & $\infty$ & $\gamma_{1}$ & $\cdots$ & $\gamma_{i}$ & $\cdots$ & $\gamma_{N / N^{\prime}-1}$ \\
\cline { 2 - 9 } 0 & 0,1 & $0,2 / N^{\prime}$ & 0,1 & 0,2 & $\cdots$ & $\cdots$ & $\cdots$ & 0,2 \\
\cline { 2 - 9 } 1 & $0, N / 2$ & $0, N / N^{\prime}$ & $0, N / 2$ & $0, N$ & $\cdots$ & $\cdots$ & $\cdots$ & $0, N$ \\
\cline { 2 - 9 }$\infty$ & 0,1 & $0,2 / N^{\prime}$ & 0,1 & 0,2 & $\cdots$ & $\cdots$ & $\cdots$ & 0,2 \\
\cline { 2 - 8 } & & &
\end{tabular}

If we desire that the previous table represent the multiplicities at the ramification points $0,1, \infty$ of a rational map, we must follow the same rules as in $\S 1$. On the other hand we recall that $N \geq 3$. It turns out that there is only one table which represents the multiplicities at the ramification points of a rational map (in particular over 1 there is just one point with multiplicity $N / N^{\prime}$ ): the table depends on the parity of $N / N^{\prime}$ (we are using observation 1.15: actually there are two tables but one comes from the composition of the other with $\frac{1}{x}$ ). It is then easy to check that connected with this table (i.e. with given multiplicities over the ramification points) there is only one diagram which represents the monodromy group of a rational covering with exactly the same multiplicities over the ramification points (cf. first part of this section). We conclude that there exists a (unique) rational map, up to homography, which pulls back the hypergeometric operator $\mathscr{H}_{N}$ into $\mathscr{H}_{N^{\prime}}$. Q.E.D.

Remark 2.16. If $N=3$, then

$$
\mathscr{N} \mathscr{C}(1,3)=\mathscr{N} \mathscr{L}(1,3) ;
$$

hence there will be exactly one nonhomographic class of Lamé operators $\mathscr{L}_{1}$ with finite monodromy of order $2 N=6$ (this is the case in [BAT]). For $N=4$, again $\mathscr{N} \mathscr{C}(1,4)=\mathscr{N} \mathscr{L}(1,4)$ and there is exactly one class: the pull-back from case 2.

Remark 2.17. In view of Remark 2.14.2, Theorem 2.15 holds not only for $n=1$ but for a general $n \in \mathbf{N}$.

Remark 2.18. It is evident that one can carry through similar calculations for every $n$ and $N$. It turns out that for every case there will be only a finite number of possibilities: in fact each Lamé operator $\mathscr{L}_{n}$ with dihedral monodromy group of order $2 N$ will be related (up to homography) to a ramified covering of $\mathbf{P}^{1}$ made by $\mathbf{P}^{1}$ of degree $n N$ : and each such covering (up to homography) is associated with a subgroup of the permutation group $\sigma_{n N}$. We can conclude that for every $n$ the number (up to homography) of Lamé operators of the type $L_{n}$ with finite monodromy group of order $2 N$ is finite. In particular, by direct computation, for $n=2$ and for $N=3$ (dihedral group of order 6), we get exactly one nonhomographic class of operators.

In general for $n \geq 1$ one can show that the number of nonhomographic Lamé operators $\mathscr{L}_{n}$ with fixed dihedral monodromy group of order $2 N$ is bounded by an increasing function of $n$.

Once we know the number of solutions of the problem $(*)$, a natural question can be posed: where are these solutions? 
In this section we study the field of definition of the coefficients of the rational map defining the pull-back for a Lamé operator with finite monodromy group, thus the field of definition of the coefficients of the Lamé operator.

Consider a Lamé operator $\mathscr{L}_{n}(1.2)$; from $e_{1}, e_{2}, e_{3}$ one can get the crossratio of $\left\{e_{1}, e_{2}, e_{3}, \infty\right\}$ (in the order)

$$
\lambda=\frac{e_{1}-e_{3}}{e_{2}-e_{3}}
$$

(invariant under homography) and the relative invariant $(\lambda \neq 0,1)$

$$
J(\lambda)=2^{8} \frac{\left(\lambda^{2}-\lambda+1\right)^{3}}{\lambda^{2}(\lambda-1)}
$$

which is invariant for homography and order.

If $\Phi$ is a rational map which gives a pull-back from a hypergeometric $\mathscr{H}_{N}$ to a Lamé operator $\mathscr{L}_{n}$ (with a certain $n \in \mathbf{N}$ ), the monodromy group of the covering induced by $\Phi$ will have cycle decompositions for the permutations which generate it given by one of the cases we have previously considered. We will indicate this case by $\kappa(\Phi)$. Remember that from $1.14 \mathcal{N}(\kappa(\Phi))$ is the number of nonhomographic coverings of $\mathbf{P}^{1}$ having the cycle decomposition of $\kappa(\Phi)$.

Proposition 3.1. If $\Phi$ gives (as a pull-back) the Lamé operator $\mathscr{L}_{n}$ with finite dihedral group of order $2 N$, it is possible to choose a homographic $\tilde{\mathscr{L}}_{n}$ given by a rational map $\tilde{\Phi}$ such that if we denote by $\mathbf{Q}(\tilde{\Phi})$ the field of the coefficients of $\tilde{\Phi}$ we have

$$
[\mathbf{Q}(\tilde{\mathbf{\Phi}}): \mathbf{Q}] \leq \alpha \cdot \mathscr{N}(\kappa(\tilde{\mathbf{\Phi}}))
$$

with $\alpha=2$, if the invariant $J$ of (the elliptic curve associated to) $\mathscr{L}_{n}$ (the same as that of $\tilde{\mathscr{L}}_{n}$ ) is $12^{3}, \alpha=3$ if $J=0$ and $\alpha=1$ otherwise.

Proof. We consider the covering given by $\Phi: \mathbf{P}^{1} \rightarrow \mathbf{P}^{1}$. If $\left\{e_{1}, e_{2}, e_{3}, \infty\right\}$ are the singular points of $\mathscr{L}_{n}$, and $J$ its invariant, one can find a homography which sends $\infty$ to $\infty$ and then if the images of $e_{1}, e_{2}, e_{3}$ are $\tilde{e}_{1}, \tilde{e}_{2}$ and $\tilde{e}_{3}$ we have that $\prod_{i=1}^{3}\left(x-\tilde{e}_{i}\right)$ is a polynomial over $\mathbf{Q}(J)$ (see Lemma 3.2). Denote by $\tilde{\Phi}$ the new rational map derived from $\Phi$ after the homography. Define $\mathbf{Q}_{s}(\tilde{\Phi})$ to be the splitting field of the coefficients of $\tilde{\Phi}\left(\mathbf{Q}_{s}(\tilde{\Phi}) \supseteq \mathbf{Q}(\tilde{\Phi})\right)$ and for every $\sigma \in \mathscr{G} a l\left(\mathbf{Q}_{s}(\tilde{\Phi}) / \mathbf{Q}\right)$, denote by $\tilde{\Phi}^{\sigma}$ the rational map obtained from $\tilde{\Phi}$ applying $\sigma$ on the coefficients:

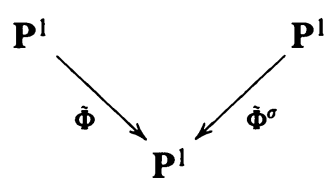

We obtain a new covering with monodromy group in the same case, i.e. the case $\kappa(\tilde{\Phi})=\kappa(\Phi)$, but in general it is not conjugate to the monodromy covering group of $\tilde{\Phi}$.

If the coverings related to $\tilde{\Phi}^{\pi}$ and $\tilde{\Phi}^{\tau}$ are isomorphic (with $\pi, \tau \in$ 
$\left.\mathscr{G} a l\left(\mathbf{Q}_{s}(\tilde{\Phi}) / \mathbf{Q}\right)\right)$, there will be a homography $\chi$ :

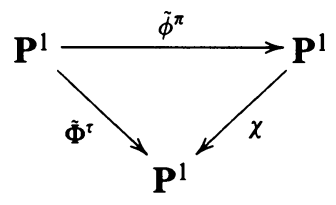

such that

$$
\chi\left(\left\{\tilde{e}_{1}^{\tau}, \tilde{e}_{2}^{\tau}, \tilde{e}_{3}^{\tau}, \infty\right\}\right)=\left\{\tilde{e}_{1}^{\pi}, \tilde{e}_{2}^{\pi}, \tilde{e}_{3}^{\pi}, \infty\right\}
$$

as sets. (In fact $\chi$ respects the multiplicity of coverings on the fibers and the points which are singular for the pull-back have peculiar multiplicities: see the cases in $\S 1$ ), and $\chi(\infty)=\infty$. In particular $J^{\tau}=J^{\pi}$ and, by our choice of $\prod\left(x-\tilde{e}_{i}\right),\left\{\tilde{e}_{i}^{\tau}, \infty\right\}=\left\{\tilde{e}_{i}^{\pi}, \infty\right\}$.

It follows that there are exactly three possibilities of constructing $\chi$ if $J=0$, two if $J=12^{3}$ and only the identity if $J$ is different. So:

$$
\left[\mathscr{G a l}_{(\mathbf{Q}}\left(\mathbf{Q}_{s}(\tilde{\mathbf{\Phi}}) / \mathbf{Q}\right): \operatorname{Fix}(\mathbf{Q}(\tilde{\boldsymbol{\Phi}}))\right] \leq \alpha \cdot \mathscr{N}(\kappa(\tilde{\boldsymbol{\Phi}})) \text {. Q.E.D. }
$$

Lemma 3.2. Consider $e_{1} \neq e_{2} \neq e_{3}$, such that $\lambda$ is the cross-ratio of $e_{1}, e_{2}, e_{3}$, $\infty$ and $J=J(\lambda)$. It is possible to find a homography $\chi$ such that $\chi(\infty)=\infty$ and

$$
\chi\left(\left\{e_{1}, e_{2}, e_{3}\right\}\right)=\left\{\tilde{e}_{1}, \tilde{e}_{2}, \tilde{e}_{3}\right\}
$$

(as sets) with $\prod\left(x-\tilde{e}_{i}\right) \in \mathbf{Q}(J)[x]$.

Proof. Consider first the case $J \neq 0,1728$. Then there exists [SI], [HU]

$$
\prod\left(x-\tilde{e}_{i}\right)=x^{3}-\frac{27}{4} \frac{J}{J-1728} x-\frac{27}{4} \frac{J}{J-1728}
$$

such that for the cross-ratio, $\tilde{\lambda}$, of $\left\{\tilde{e}_{1}, \tilde{e}_{2}, \tilde{e}_{3}, \infty\right\}$ we have $J(\tilde{\lambda})=J$. The existence of the prescribed homography follows after a permutation of the $\tilde{e}_{i}$ 's. If $J=1728$, we have $\lambda=-1, \frac{1}{2}, 2:$ it is enough to take the Legendre form $0,1, \lambda$. Finally for $J=0, \lambda=-\omega,-\omega^{2}$ where $\omega^{3}=1$ and our $\left\{e_{2}, \infty\right\}$ is homographically connected with $\left\{-1,-\omega,-\omega^{2}, \infty\right\}$. Q.E.D.

Remark 3.3. Note that for $\mathscr{N}(\kappa(\phi))=1$, necessarily $J \in \mathbf{Q}$.

\section{4}

Examples. We want to give some examples of Lamé operators with prescribed monodromy group.

4.1. If $n=1$ and $N=3$, the monodromy group is of order $6=2 N$; by our calculations there is only one Lamé operator with monodromy group of order 6 (up to homography, from 5). This agrees with the work of F. Baldassarri [BAT] (see observation 1.14).

4.2. If $n=1$ and $N=4$, the situation is like the previous one. There is only one Lamé operator (up to homography) with dihedral group of order 8 (deriving from the cases 2). So it is enough to calculate a pull-back mapping in each case 2 or, equivalently (by observation 1.15) in $2 \uparrow$. In the case $2 \uparrow$ the general equation for the pull-back map (in $\mathscr{C}(1,4))$ is $(x$ for the variable in $\mathscr{L}$ and $x^{\prime}$ for $\left.\mathscr{H}\right)$ :

$$
\frac{A\left(x-e_{1}\right)}{\left(x-e_{2}\right)\left(x-e_{3}\right)(x-\lambda)^{2}}=-\frac{(x-\xi)^{4}}{\left(x-e_{2}\right)\left(x-e_{3}\right)(x-\lambda)^{2}}+1
$$


( $\infty$ is sent to 0 with multiplicity $3, e_{1} \neq e_{2} \neq e_{3} \neq \xi \neq \lambda$ ). It follows that $J=0,12^{3}$ does not happen. So, by Proposition 3.1 there will be a pull-back mapping defined in $\mathbf{Q}$; in fact

$$
x^{\prime}=\frac{-4\left(x-\frac{7}{4}\right)}{\left(x^{2}+2\right)(x-2)^{2}}=-\frac{(x-1)^{4}}{\left(x^{2}+2\right)(x-2)^{2}}+1 .
$$

Of course for 2 the pull-back is given by composing with $x^{\prime \prime}=1 / x^{\prime}$.

It follows that the only elliptic curve on which there is defined a Lamé operator $n=1$ with dihedral monodromy group of order 8 is

$$
y^{2}=4\left(x-\frac{7}{4}\right)\left(x^{2}+2\right) \text {. }
$$

4.3. If $n=1$ and $N=5$ (dihedral group of order 10), there are two groups for the coverings (up to homography): one from each of 4 and 5. Hence there are two classes of Lamé operators.

So in every case it is enough to find a pull-back mapping in order to know the others by homography. By 4 the rational map has to satisfy

$$
\begin{gathered}
\frac{\left(x-e_{1}\right)\left(x-\lambda_{1}\right)^{2}\left(x-\lambda_{2}\right)^{2}}{A\left(x^{2}+p x+q\right)}=\frac{(x-\xi)^{5}}{A\left(x^{2}+p x+q\right)}+1, \\
x^{\prime}=\frac{(x+3)\left(x^{2}-4 x+9\right)^{2}}{40\left(x^{2}-\frac{14}{4} x+\frac{244}{40}\right)}=\frac{(x-1)^{5}}{40\left(x^{2}-\frac{14}{4} x+\frac{244}{40}\right)}+1
\end{gathered}
$$

( $\infty$ is sent to $\infty$ with multiplicity 3 ). Of course $x^{\prime \prime}=1 / x^{\prime}$ for $4 \uparrow$.

And for $5 \uparrow$

$$
\frac{A(x-\lambda)^{2}}{\left(x^{3}+p x+q\right)(x-\theta)^{2}}=-\frac{(x-\xi)^{5}}{\left(x^{3}+p x+q\right)(x-\theta)^{2}}+1
$$

we find the solution

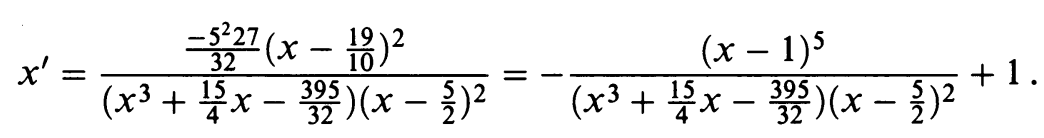

Obviously for $5 x^{\prime \prime}=1 / x^{\prime}$. It follows that the only two elliptic curves where a Lamé operator with monodromy group of order 10 is defined are

$$
y^{2}=\left(x^{2}-\frac{7}{2} x+\frac{61}{10}\right)(x+3), \quad y^{2}=x^{3}+\frac{15}{4} x-\frac{395}{32} .
$$

4.4. In Remark 2.16 we have noticed that for $n=2$ there is exactly one class of Lamé operators in $L_{2}$ with monodromy group dihedral of order 6 (from 2). To find a representative it is enough to construct a pull-back for 2 . This rational map must satisfy

$$
\frac{\left(x^{2}+p x+q\right)\left(x^{2}+\lambda_{1} x+\lambda_{2}\right)^{2}}{A\left(x-e_{3}\right)}=\frac{\left(x^{2}+r x+t\right)^{3}}{A\left(x-e_{3}\right)}+1
$$

( $\infty$ is sent into $\infty$ with multiplicity 5). We get the rational map

$$
x^{\prime}=\frac{\left(x^{2}+8 x+\frac{148}{9}\right)\left(x^{2}+2 x+\frac{4}{9}\right)^{2}}{\frac{-64}{9}\left(x+\frac{1}{3}\right)}=\frac{\left(x^{2}+4 x+\frac{16}{9}\right)^{3}}{\frac{-64}{9}\left(x+\frac{1}{3}\right)}+1 .
$$


Thus, the only elliptic curve in which a Lamé operator of the type $\mathscr{L}_{2}$ with finite dihedral monodromy group of order 6 is defined is

$$
y^{2}=\left(x^{2}+8 x+\frac{148}{9}\right)\left(x+\frac{1}{3}\right) \text {. }
$$

We now apply our method to study Lamé operators with infinite monodromy group.

This section is divided into two parts. In the first part we will study rational pull-backs of hypergeometric operators giving Lamé operators of type $\mathscr{L}_{-\frac{1}{2}}$. We will see, in particular, that there are only four classes of operators of the type $\mathscr{L}_{-\frac{1}{2}}$ which are rational pull-backs (cf. [CH-CH1, 2]). In the second part we deal with the notion of a general second order differential equation which is an algebraic pull-back of hypergeometric operators (i.e. the pull-back is made by an algebraic map): we will give a bound for the degree of such a map.

As we said, we wish to deal with Lamé operators $\mathscr{L}_{-\frac{1}{2}}$ which are rational pull-backs of hypergeometric operators.

We state the problem in a slightly different way.

Namely, in this part of the section, we want to study all the second order differential operators $\mathscr{L}$ with four singular points and with Riemann data

$$
\left(\begin{array}{cccc}
e_{1} & e_{2} & e_{3} & \infty \\
0 & 0 & 0 & 1 \\
0 & 0 & 0 & 1
\end{array}\right)
$$

(Note that the previous Riemann data are those of a $\mathscr{L}_{-\frac{1}{2}}$ after the Halphen transformation) which are pull-backs by a rational map of a hypergeometric operator. Necessarily the hypergeometric operator will be of the type $\mathscr{H}_{\lambda_{0}, \lambda_{1}, \infty}$ $\left(\lambda_{0}, \lambda_{1} \in \mathbf{N} \cup\{\infty\}\right.$ and with exponent difference $\frac{1}{\lambda_{0}}, \frac{1}{\lambda_{1}}$ and 0$)$. The formula for the degree, $M$, of such rational map $\Phi$ is

$$
M\left(\frac{1}{\lambda_{0}}+\frac{1}{\lambda_{1}}-1\right)=-2
$$

And the number of ramification points (i.e. the number of points in the set $\Gamma=\Phi^{-1}\{0,1, \infty\}$ (cf. $\left.\S 1\right)$ :

$$
M\left(\frac{1}{\lambda_{0}}+\frac{1}{\lambda_{1}}\right)=\# \Gamma-4
$$

If $\lambda_{0}=\lambda_{1}=\infty, M=2$.

Otherwise, from the table rules, $M$ must be greater than each finite $\lambda_{0}$ and $\lambda_{1}$. In fact (we suppose that if one of $\lambda_{i}$ is $\infty$, then it is $\lambda_{1}$ ) take $\xi \in$ $\Gamma \backslash\left\{e_{1}, e_{2}, e_{3}, \infty\right\}$ : in $\xi$ the operator $\mathscr{L}$ is nonsingular. It follows that the exponent difference in $\xi$ is 1 . By $1.3 \xi$ cannot belong to the fiber over $\infty$ (and, if $\lambda_{1}=\infty$, it cannot be over 1 ). The only possibility is that $\xi$ is sent in 0 or 1 and with multiplicity $\lambda_{0}$ or $\lambda_{1}$ (or, in the case $\lambda_{1}=\infty$, it is sent to 0 , with multiplicity $\left.\lambda_{0}\right)$. By the first table rule in $\S 1$ the multiplicity of $\Phi$ in every point must be less than or equal to the degree $M$ (note that in the case with $\lambda_{0}, \lambda_{1}$ both finite necessarily there will be one $\xi$ as above in each fiber over 0 and 1 ). 
It is possible to bound the values of $M, \lambda_{0}$ and $\lambda_{1}$.

From (5.1) putting $\lambda_{0} \leq \lambda_{1}: \lambda_{0}=\lambda, \lambda_{1}=\lambda+n, n \in \mathbf{N}$, one gets

$$
\frac{1}{\lambda}+\frac{1}{\lambda+n}=\frac{M-2}{M} .
$$

It follows that $\lambda=\lambda_{0} \geq 2$. Suppose now $M>4$; then

$$
\frac{1}{2}<\frac{M-2}{M}<1 .
$$

So by the previous equality

$$
\frac{1}{\lambda}+\frac{1}{\lambda+n}>\frac{1}{2} .
$$

If we suppose also that $\lambda>2$, we get the condition

$$
\frac{4 \lambda-\lambda^{2}}{\lambda-2}>0
$$

and $\lambda>4$. So there are not pull-backs for $M>4$ and $\lambda \geq 4$.

If $\lambda=4$, the equation (5.1) will be

$$
\frac{1}{4}+\frac{1}{4+n}=\frac{M-2}{M} .
$$

For $M=4$ there is the solution $n=0$. If $M>4$ from

$$
\frac{1}{4}+\frac{1}{4+n}=\frac{M-2}{M}>\frac{1}{2}
$$

we see that it is impossible to find solutions.

In conclusion one admissible triple will be: $\lambda_{0}=\lambda_{1}=4$ and $M=4$, and we have found that it is impossible to have rational functions with $M>4$ and $\lambda=\lambda_{0} \geq 4$. By previous observation $\lambda_{0} \leq M$, so it follows that the only possible cases are $\lambda_{0}=2,3$.

If $\lambda_{0}=\lambda=2,(5.1)$ will be

$$
\frac{1}{2}+\frac{1}{2+n}=\frac{M-2}{M}
$$

and admissible cases are

\begin{tabular}{|c|c|c|c|c|c|c|}
\hline & & & & $\lambda_{0}$ & $\lambda_{1}$ & $M$ \\
\hline$\lambda_{0}$ & $\lambda_{1}$ & $M$ & \# & 2 & 3 & 12 \\
\hline$\infty$ & $\infty$ & 2 & 4 & 2 & 4 & 8 \\
\hline C & $\infty$ & 3 & 2 & 2 & 6 & 6 \\
\hline 2 & $\infty$ & 4 & 3 & 3 & 3 & 6 \\
\hline & & & & 4 & 4 & 4 \\
\hline
\end{tabular}

$$
\begin{array}{lll}
\lambda_{0}=2, & \lambda_{1}=3, & M=12, \\
\lambda_{0}=2, & \lambda_{1}=4, & M=8, \\
\lambda_{0}=2, & \lambda_{1}=6, & M=6 .
\end{array}
$$

For $\lambda_{0}=3$

$$
\frac{1}{3}+\frac{1}{3+n}=\frac{M-2}{M}
$$

and the only admissible case is $n=0$, i.e. $\lambda_{0}=\lambda_{1}=3, M=6$. Finally the only admissible cases are: 
For every admissible triple above one can construct the possible tables, and for every table the related nonconjugate groups (connected groups). By a combinatorial argument the number of nonconjugate groups (= not homographic coverings of $\mathbf{P}^{1}$ with given ramification data $=$ nonhomographic rational functions which transform by pull-back a hypergeometric operator $\mathscr{H}_{\lambda_{0}, \lambda_{1}, \infty}$ into a Lamé with Riemann data as in (5.0)) is presented by \# in Table (5.2).

We note that to every table there corresponds one and only one group (not as in $\S 2$ where a given table corresponds to many nonconjugate groups). On the other hand it may happen that the same Lamé operator is presented as rational pull-back by two nonhomographic rational functions.

It turns out that all the hypergeometric operators in Table (5.2) are related to the operator $(\infty, 2,3)$; i.e., they can be derived from $(\infty, 2,3$,$) by a rational$ transformation. It follows that each covering arising from Table (5.2) is a factor of one connected with $(\infty, 2,3)$. (This is consistent with the degree of the coverings. The only problem arises from $(\infty, 2,4)$ which admits coverings of degree 8 (which does not divide 12 , the degree of $(\infty, 2,3)$ coverings). But in this case $(\infty, 2,4)$ is transformed by means of a quadratic transformation $((22)$, p. 112 in [BATE]) in $(\infty, 2, \infty):$ the number of coverings related to these two operators is the same. A direct computation of the cycle decomposition of the fibers shows that every covering of $(\infty, 2,4)$ can be factorized by the rational map which connects $(\infty, 2, \infty)$ and $(\infty, 2,4)$. On the other hand $(\infty, 2, \infty)$ is a cubic transform of $(\infty, 2,3)((43)$, p. 114 in [BATE]).)

So it is enough to study the coverings of $(\infty, 2,3)$. But the hypergeometric equation which is related,

$$
F\left(\frac{1}{12}, \frac{5}{12}, 1, z\right)
$$

arises from the family of elliptic curves having parameter equal to the $j$ invariant. Our coverings can be interpreted as stable families of elliptic curves with four singular fibers. In this sense the problem has been studied by [BE] (the article which gave us the idea for the present work).

In a private letter to Dwork, Beukers signals that the six families of elliptic curves give only four nonequivalent Lamé operators of the prescribed type. (On the other hand they are connected with the Apery examples [BE-ST].)

N. B. This agrees with [CH-CH1, 2].

In this second part of the section we deal with pull-backs of hypergeometric operators realized by algebraic functions.

Take a hypergeometric operator

$$
\mathscr{H}=D_{x}^{2}+\left(\frac{1-\lambda}{x}+\frac{1-v}{x-1}\right) D_{x}+\frac{(1-\lambda-v+\eta)(1-\lambda-v-\eta)}{4 x(x-1)}
$$

with Riemann data:

$$
\left(\begin{array}{ccc}
0 & 1 & \infty \\
0 & 0 & \frac{1}{2}(1-\lambda-v+\eta) \\
\lambda & v & \frac{1}{2}(1-\lambda-v-\eta)
\end{array}\right)
$$

and suppose that the pull-back, made by an algebraic map $x=x(t)$, is rational in $t$, i.e.

$$
x^{*}(\mathscr{H}) \in \mathbf{C}(t)\left[D_{t}\right] .
$$


Without restrictions one can think that $x(\infty)=\infty$ and that $\infty$ is a singular point for $x^{*}(\mathscr{L})$. We claim:

Proposition 5.5. In the previous hypothesis, the algebraic relationship (polynomial in $x$ and $t$ )

$$
P(x, t)=0
$$

is at most of degree two in $x$ and the degree of $t$ is controlled by the number $k+1$ of singularities of $x^{*}(\mathscr{H})$ and by the number $N+1$ of points over $\infty$ in the covering given by $x=x(t)$.

Proof. It is just a computation to show that in $x^{*}(\mathscr{C})$ every point over $\mathbf{P}^{1} \backslash\{\infty\}$ will have exponents of the type:

$$
0, q
$$

( $q=1$ if the point is not singular, $q \neq 1$ otherwise).

For the points on the fiber over $\infty, x^{-1}(\infty) \quad\left(\# x^{-1}(\infty)=N+1\right)$, this is not true. In order to have one exponent equal to zero in each $a_{j} \in x^{-1}(\infty) \backslash\{\infty\}$, one has to twist $x^{*}(\mathscr{H})$ by a simple function of the type

$$
G=\prod_{a_{j} \in x^{-1}(\infty) \backslash\{\infty\}}\left(t-a_{j}\right)^{A_{j}}
$$

for appropriate $A_{j} \in \mathbf{Q}$. Finally, by this modification of exponents, the operator

$$
\frac{1}{G} \circ x^{*}(\mathscr{H}) \circ G
$$

will have the following structure (Heun):

$$
D_{t}^{2}+\left(\sum_{j=1}^{k} \frac{c_{j}}{\left(t-b_{j}\right)}\right) D_{t}+\frac{\alpha \beta x^{k-1}+\cdots}{\prod_{j}\left(t-b_{j}\right)}
$$

(with $\alpha, \beta$ exponents at $\infty$, satisfying the fuchsian relationship). On the other hand $\frac{1}{G} \circ x^{*}(\mathscr{H}) \circ G$ is equal (by its genesis) to:

$$
\begin{aligned}
D_{t}^{2}+ & \left(\frac{2 G^{\prime}}{G}+\left(\frac{1-\lambda}{x}+\frac{1-v}{x-1}\right) x^{\prime}-\frac{x^{\prime \prime}}{x^{\prime}}\right) D_{t} \\
& +\frac{(1-\lambda-v)^{2}-(\eta)^{2}}{4 x(x-1)} x^{\prime 2}+\frac{G^{\prime \prime}}{G}+\left(\left(\frac{1-\lambda}{x}+\frac{1-v}{x-1}\right) x^{\prime}-\frac{x^{\prime \prime}}{x^{\prime}}\right) \frac{G^{\prime}}{G}
\end{aligned}
$$

(where the derivations are $t$-derivations). Making the equality between (5.6) and (5.7), one finds:

$$
\sum_{j=1}^{k} \frac{c_{i}}{\left(t-b_{i}\right)}=\frac{2 G^{\prime}}{G}+\left(\frac{1-\lambda}{x}+\frac{1-v}{x-1}\right) x^{\prime}-\frac{x^{\prime \prime}}{x^{\prime}}
$$

and

$$
\frac{(1-\lambda-v)^{2}-\eta^{2}}{4 x(x-1)} x^{\prime 2}=-\frac{G^{\prime \prime}}{G}-\left(\left(\frac{1-\lambda}{x}+\frac{1-v}{x-1}\right) x^{\prime}-\frac{x^{\prime \prime}}{x^{\prime}}\right) \frac{G^{\prime}}{G}+\frac{\alpha \beta x^{k-1}+\cdots}{\prod\left(t-b_{i}\right)} .
$$

Using (5.8), the right side of (5.9) will be a rational function, $S(t)$, in $t$ : its degree will be in general, $2 N+k+1$ at denominator and $2 N+k$ at numerator. 
Integrating (5.8) one obtains

$$
\prod_{i=1}^{k}\left(t-b_{i}\right)^{c_{i}}=\frac{C G^{2} x^{1-\lambda}(x-1)^{1-v}}{x^{\prime}}
$$

Multiplying (5.10) by the square root of (5.9) we get

$$
\left(\frac{(1-\lambda-v)^{2}-\eta^{2}}{4 x(x-1)}\right)^{\frac{1}{2}} C G^{2} x^{1-\lambda}(x-1)^{1-v}=S(t)^{\frac{1}{2}} \prod\left(t-b_{i}\right)^{c_{i}}
$$

Taking the logarithmic derivative we have

$$
\left(\frac{1-\lambda-\frac{1}{2}}{x}+\frac{1-v-\frac{1}{2}}{x-1}\right) x^{\prime}=\frac{1}{2} \frac{S^{\prime}(t)}{S(t)}+\sum \frac{c_{i}}{\left(t-b_{i}\right)}-\frac{2 G^{\prime}}{G} .
$$

Dividing by the square root of (5.9) and squaring gives

$$
\begin{gathered}
\left(\frac{\left(1-\lambda-\frac{1}{2}\right)(x-1)+\left(1-v-\frac{1}{2}\right) x}{x(x-1)}\right)^{2} \frac{4 x(x-1)}{(1-\lambda-v)^{2}-\eta^{2}} \\
=\left(\frac{1}{2} \frac{S^{\prime}(t)}{S(t)}+\sum \frac{c_{i}}{\left(t-b_{i}\right)}-\frac{2 G^{\prime}}{G}\right)^{2} \frac{1}{S(t)} .
\end{gathered}
$$

From formula (5.11), viewed as an algebraic relationship between $t$ and $x$, one obtains the desired conclusion. Q.E.D.

N. B. In the equation (5.11) the right side has denominator of degree $6 N+3 k$ in $t$, which is a bound for the degree of the numerator, too.

\section{BIBLIOGRAPHY}

[BA] F. Baldassarri, On algebraic solutions of Lamé differential equation, J. Differential Equations 41 (1981), 44-58.

[BA1] - On second order linear differential equations on algebraic curves, Amer. J. Math. 102 (1980), 517-535.

[BAT] _ Towards a Schwarz list for Lamé differential operators via division points on elliptic curves, preprint.

[BA-DW] F. Baldassarri and B. Dwork, On second order differential equations with algebraic solutions, Amer. J. Math. 101 (1979), 42-76.

[BATE] H. Bateman (manuscript project), Higher transcendental functions, McGraw-Hill, 1953.

[BE] A. Beauville, Les familles stables de courbes elliptiques sur $\mathbf{P}^{1}$ admettant quatre fibres singulieres, C. R. Acad. Sci. Paris 294 (1982), 657-660.

[BE-ST] F. Beukers and J. Stienstra, On the Picard-Fuchs equation and the formal Brauer group of certain elliptic K3-surfaces, Math. Ann. 271 (1985), 269-304.

[BE-HE] F. Beukers and G. Heckman, Monodromy for the hypergeometric function ${ }_{n} F_{n-1}$, Invent. Math. 95 (1989), 325-354.

[CH-CH] D. V. Chudnovsky and G. V. Chudnovsky, Application of Pade approximations to the Grothendieck conjecture on linear differential equations, Lecture Notes in Math., vol. 1135, Springer-Verlag, pp. 83-84.

[CH-CH1] _ Transcendental methods and theta-functions, Proc. Sympos. Pure Math., vol. 49, part 2, Amer. Math. Soc., Providence, RI, 1989, pp. 167-232.

[CH-CH2] _ A random walk in higher arithmetic, Adv. Appl. Math. 7 (1986), 101-122.

[CO] F. Coppi, Tesi di laurea, Universitá di Padova, 1992. 
[DW] B. Dwork, Arithmetic theory of differential equations, Sympos. Math., vol. 24, Academic Press, London, 1981, pp. 225-244.

[DW1] _ Differential operators with nilpotent p-curvature, Amer. J. Math. (to appear).

[FO] O. Forster, Lectures on Riemann surfaces, Graduate Texts in Math., 81, Springer-Verlag, New York and Berlin, 1977.

[HO] T. Honda, Algebraic differential equations, Sympos. Math., vol. 24, Academic Press, London, 1981, pp. 169-204.

[HU] D. Husemöller, Elliptic curves, Springer-Verlag, 1987.

[KA] N. Katz, Algebraic solutions of differential equations; p-curvature and the Hodge filtration, Invent. Math. 18 (1972), 1-118.

[KA1] , Nilpotent connections and the monodromy theorem, Publ. Math. Inst. Hautes Études Sci. 39 (1971), 355-412.

[KA2] _ A conjecture in the arithmetic theory groups, Invent. Math. 87 (1987), 13-61.

[KI] T. Kimura, On fuchsian differential equations reducible to hypergeometric equations by linear transformations, Funkcial. Ekvac. 13 (1970), 213-232.

[MA] W. S. Massey, A basic course in algebraic topology, Graduate Texts in Math., 127, SpringerVerlag, New York and Berlin, 1991.

[PL] E. Poole, Introduction to the theory of linear differential equations, Oxford, 1936.

[SE] L. Greenberg, Maximal fuchsian groups, Bull. Amer. Math. Soc. 69 (1963), 569-573.

[SE-TR] H. Seifert and W. Threlfall, A textbook of topology, Academic Press, New York, 1980.

[SI] J. Silverman, The arithmetic of elliptic curves, Graduate Texts in Math., 106, SpringerVerlag, New York and Berlin, 1986.

[SIN] M. F. Singer, Algebraic solutions of nth order linear differential equations, Proc. Queen's Number Theory Conf. 1979, Queen's Paper in Pure and Appl. Math., 54, Queen's Univ. Kingston, 1980, pp. 379-420.

[TR] M. Tretkoff, Algebraic extentions of the field of rational functions, Comm. Pure Appl. Math. 24 (1979), 491-497.

[WW] E. T. Whittaker and G. N. Watson, $A$ course in modern analysis, Cambridge Univ. Press, 1927.

Dipartimento di Matematica Pura e Applicata Universitá di Padova, Via Belzoni 7, 35131 Padova, Italy

E-mail address: chiarellot to@pdmat1.math.unipd.it 